\title{
Breaking Dark Matter Degeneracies with Neutrino Detectors
}

\author{
Sergio Palomares-Ruiz* ${ }^{\dagger}$ \\ IPPP, Department of Physics, Durham University, Durham DH1 3LE, United Kingdom \\ and \\ Centro de Física Teórica de Partículas, Instituto Superior Técnico, 1049-001 Lisboa, Portugal \\ E-mail: sergio.palomares.ruiz@ist.utl.pt
}

\begin{abstract}
In this talk we show how future neutrino detectors could be used to break some of the degeneracies in the dark matter (DM) parameter space and to test some of the DM properties. We consider first the case of annihilations inside the Sun and show how detectors with good neutrino energy resolution could help to discriminate between different annihilation channels (soft or hard) and break their degeneracy with the DM-proton elastic scattering cross section. Then, we consider DM annihilations and decays in the galactic halo. By making use of the fact that neutrinos are the least detectable particles of the Standard Model and assuming DM only annihilates or decays into neutrinos, we show how future neutrino detectors could be great tools to obtain general limits on the total DM annihilation cross section and on the DM lifetime.
\end{abstract}

10th International Workshop on Neutrino Factories, Super beams and Beta beams

June 30 - July 52008

Valencia, Spain

\footnotetext{
* Speaker.

†The author is supported by the Portuguese Grant POCI/FP/81919/2007 of the FCT and partially by the Spanish Grant FPA2005-01678 of the MCT.
} 
Introduction. - The next generation of neutrino experiments will bring us to the era of precision measurements in neutrino physics and may allow us to (totally or partially) break the so-called neutrino degeneracies. Nevertheless and in addition to all their capabilities to solve the degeneracy problem in the neutrino parameter space, present and future detectors, could also be good tools for other purposes. Among the possible synergies of these detectors, they could be used to break some of the degeneracies in the dark matter (DM) parameter space and to test some of the DM properties.

In this talk we consider three different possibilities for the origin of the studied neutrinos: from DM annihilations in the center of the Sun and from annihilations and decays in our galactic halo. In the first case we show that by using the spectral information provided by future neutrino detectors some of the DM properties could eventually be reconstructed, as the type of the DM annihilation channel (soft or hard) and the DM-proton elastic scattering cross section [1]. In the other two cases, we show how we could test some other DM properties, as the total DM annihilation cross section [2, 3, 4] and the DM lifetime [5].

DM annihilations in the Sun. - Indirect signals of the existence of DM are expected to be stronger if they come from places where DM may accumulate, as the center of the Sun. In this case, high-energy neutrinos would be produced in the DM annihilation either directly or by subsequent decay of Standard Model (SM) particles.

Searches of these high-energy neutrinos have been performed in Čerenkov neutrino detectors with no positive evidence found so far. Hence, stringent bounds have already been set [6] and will be further improved by neutrino telescopes [7]. However, these experiments can only provide limited information on the neutrino spectrum as they are not able to reconstruct the neutrino energy. Here and following Ref. [1], we restrict our analysis to Magnetized Iron Calorimeter Detectors (MIND) [8]. These detectors will not only measure precisely the energy and direction of neutrinoinduced muons (and possibly of electrons [8]), but also the energy and angle of the hadron shower, so that a good energy and direction resolution for the incoming neutrino will be achieved. Here we show how the use of the neutrino spectra could help to break the degeneracy between the DMproton elastic scattering cross section and the branching ratios into soft or hard channels.

As the muon energy resolution for these detectors gets worse at high energies, we only consider light $\mathrm{DM}$ candidates with masses below $\sim 80 \mathrm{GeV}$ and therefore, we focus on its dominant annihilation modes for these masses, i.e. $\tau^{+} \tau^{-}$(hard) and $b \bar{b}$ (soft) channels. Note also that due to the relative "small" size of these detectors (as compared with neutrino telescopes) and the very strong bounds on spin-independent cross sections coming from direct searches [9], we cannot consider the case of DM annihilations in the Earth, as the abundance of nuclei with odd mass number is extremely small. This is the reason why we focus on DM annihilations in the Sun, for which the much more weakly constrained spin-dependent cross section [10] may play the dominant role.

We compute the number of electron and muon neutrino-induced charged-current events for a DM mass of $50 \mathrm{GeV}$, by using the evolved neutrino spectra from Ref. [11]. We conservatively consider nine 5-GeV energy bins, where the low energy cut is adopted to avoid the atmospheric neutrino background. The expected number of muon (electron) neutrino-induced events in each bin is computed as a function of the DM-proton elastic scattering cross section and of the branching ratios into $b^{+} b^{-}$(soft channel) and $\tau^{+} \tau^{-}$(hard channel) and is then fitted performing a $\chi^{2}$ analysis. For further details of this analysis we refer the reader to Ref. [1]. 

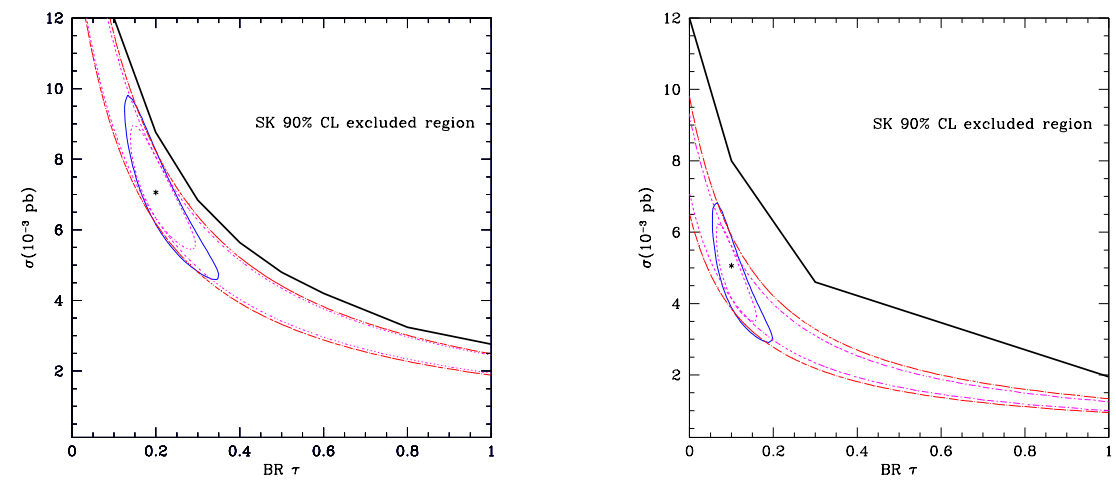

Figure 1: Left panel: The solid blue (dotted-dashed red) contours denote the 90\% CL limits, for 2 degrees of freedom, for the simultaneous extraction of the DM-proton cross section and the annihilation branching ratio into the $\tau^{+} \tau^{-}$ channel with (without) energy information by using only fully-contained events with an exposure of $750 \mathrm{kton} \cdot \mathrm{yrs}$. The results are for a DM mass of $50 \mathrm{GeV}, \sigma=7 \times 10^{-3} \mathrm{pb}$ and $\mathrm{BR}_{\tau^{+} \tau^{-}}=20 \%$. The dashed magenta lines (inner contours) illustrate the case (for $90 \% \mathrm{CL}$ ) in which the atmospheric neutrino background is integrated only over the real size of the Sun. We also show the $90 \%$ CL excluded region by Super-Kamiokande [6]. Right panel: Same for a DM mass of $70 \mathrm{GeV}, \sigma=5 \times 10^{-3} \mathrm{pb}$ and $\mathrm{BR}_{\tau^{+} \tau^{-}}=10 \%$. From Ref. [1].

In the left (right) panel of Fig. 1 we show the results for a DM mass of $50 \mathrm{GeV}(70 \mathrm{GeV})$ and the input values are $\sigma=7 \times 10^{-3} \mathrm{pb}\left(5 \times 10^{-3} \mathrm{pb}\right)$ and $\mathrm{BR}_{\tau^{+} \tau^{-}}=20 \%(10 \%)$. We have assumed $750 \mathrm{kton} \cdot$ years of exposure and have only included electron-like and contained muon-like events. The dotted-dashed red lines depict the $90 \%$ confidence level (CL) contours assuming that no energy information is available: a larger annihilation rate could always be traded by a lower branching ratio into a hard channel. Note that there is a continuous region of degenerate solutions, and thus it is not possible to extract either the branching ratios or the DM-proton elastic scattering cross section. Instead, if we include the information on the neutrino energy spectrum, we obtain the $90 \% \mathrm{CL}$ contours depicted by the solid blue line. The strong correlation between $\sigma$ and $\mathrm{BR}_{\tau^{+} \tau^{-}}$ is broken: the differential neutrino spectrum for each possible annihilation channel (hard or soft) has a characteristic shape, different from one channel to another. We also illustrate the results for the ideal case for which the atmospheric neutrino background only contributes within the real angular size of the Sun (dashed magenta lines). As can be seen, by considering a more detailed and realistic angular resolution than that used (see Ref. [1]), our results would not significantly improve.

DM annihilations and decays in the galactic halo.- We consider now DM annihilations and decays in our galactic halo and show how the null signal in present neutrino detectors allow us to obtain very stringent and general bounds on the total DM annihilation cross section and DM lifetime. The idea [2] is to assume that DM annihilates [2, 3, 4] or decays [5] only into neutrinos, so a limit on their flux, conservatively and in a model-independent way, would set an upper (lower) bound on the DM annihilation cross section (lifetime). This is so because neutrinos are the least detectable particles in the SM and hence any other possible annihilation (decay) mode would produce gamma rays, which are much easier to detect, and would allow one to set a much stronger (but model-dependent) bound. Let us stress that this assumption is not related to a particular and realistic case. Nevertheless and for the reasons just stated, it is valid for any generic model, in which DM annihilates (decays) into at least one SM particle. Therefore, these bounds represent limits 

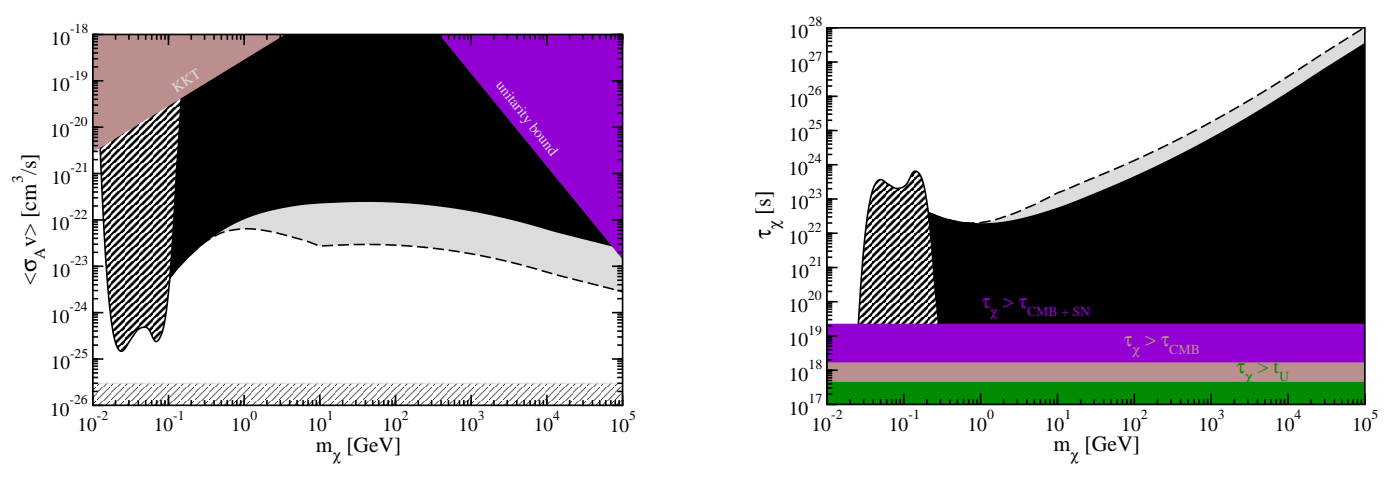

Figure 2: Bounds on the total DM annihilation cross section (left panel) and DM lifetime (right panel) for a wide range of DM masses $\left(m_{\chi}\right)$ obtained using different approaches: full-sky signal (dark area), angular signal (light area) and $90 \%$ CL limit using SK data at low energies [12] (hatched area). Other bounds are also shown. Left panel: the unitarity bound [13], the limit above which the cusps of the DM halos are too flat (KKT) [14] and the natural scale for thermal relics. Right panel: bounds from Cosmic Microwave Background (CMB) observations [16] and CMB plus Supernovae data [17] (both at $2 \sigma \mathrm{CL}$ ) and the line $\tau_{\chi}=t_{U}$, with $t_{U} \simeq 4 \times 10^{17} \mathrm{~s}$ the age of the Universe. From Ref. [19].

on the total annihilation cross section (lifetime) of the DM candidate and not only on its partial annihilation cross section (lifetime) due to the annihilation (decay) channel into neutrinos. Even in this conservative case, stringent bounds can be obtained by comparing the expected time-integrated annihilation signal of all galactic halos [2] and the signal from annihilations [3, 4] or decays [5] in the Milky Way Halo with the background at these energies. The constraints so obtained are more restrictive than other general limits [13, 14, 15, 16, 17] (however, see also [18]).

For $E_{v} \gtrsim 100 \mathrm{MeV}$, the main source of background for a possible neutrino signal from DM annihilations or decays is the flux of atmospheric neutrinos. In this energy range, we follow the approach of Ref. [3] where we refer the reader for details. We obtain a general bound by comparing the $\left(v_{\mu}+\bar{v}_{\mu}\right)$ neutrino flux from DM annihilation (decays) in the halo with the corresponding atmospheric neutrino flux in an energy bin of width $\Delta \log _{10} E_{v}=0.3$ around $E_{v}=m_{\chi}\left(E_{v}=m_{\chi} / 2\right)$. The most conservative bound is obtained with the full-sky signal, and this is shown in both panels of Fig. 2 where the dark areas represent the excluded regions. However, a better limit can be obtained if we consider instead a field of view with a half-angle cone of $30^{\circ}\left(30^{\circ} \times \sqrt{10 \mathrm{GeV} / E_{v}}\right)$ for neutrinos with energies above (below) $10 \mathrm{GeV}$. This limit is shown in both panels of Fig. 2 by the dashed lines (light areas), which improves upon the previous case by a factor of a few.

As follows from these results, it is expected that a more detailed analysis should give more stringent limits. We now show the results of a more careful treatment of the energy resolution and backgrounds from Refs. [4, 5] (where we refer the reader for details). They substantially improve (and extend) these limits in the energy range $15 \mathrm{MeV} \lesssim E_{v} \lesssim 130 \mathrm{MeV}$ where the best data comes from the search for the diffuse supernova background by the Super-Kamiokande (SK) detector which has looked at positrons in the energy interval $18 \mathrm{MeV}-82 \mathrm{MeV}$ [12]. By performing a $\chi^{2}$ analysis, analogous to that of the SK collaboration [12], we compare the shape of the background spectrum (the atmospheric $v_{e}$ and $\bar{v}_{e}$ flux and the Michel electrons and positrons from the decays of sub-threshold muons) to that of the signal, and we extract the limits on the DM annihilation cross section [4] and DM lifetime [5]. These 90\% CL bounds are shown in both panels of Fig. 2 by the hatched areas and they clearly improve (and extend to lower masses) by about an order of magnitude upon the general and very conservative bound for higher energies. 
Conclusions. - In this talk we have followed two different approaches [1, 2, 3, 4, 5, 19] to show how neutrino detectors can be used to break DM degeneracies and to test some of the DM properties. They could be great tools to discriminate between different DM annihilation channels (soft or hard), measure (or limit) the spin-dependent DM-proton elastic scattering cross section and obtain model-independent bounds on the total DM annihilation cross section and DM lifetime.

\section{References}

[1] O. Mena, S. Palomares-Ruiz and S. Pascoli, Phys. Lett. B 664 (2008) 92 [arXiv:0706.3909 [hep-ph]].

[2] J. F. Beacom, N. F. Bell and G. D. Mack, Phys. Rev. Lett. 99 (2007) 231301 [arXiv:astro-ph/0608090].

[3] H. Yuksel, S. Horiuchi, J. F. Beacom and S. Ando, Phys. Rev. D 76 (2007) 123506 [arXiv:0707.0196 [astro-ph]].

[4] S. Palomares-Ruiz and S. Pascoli, Phys. Rev. D 77 (2008) 025025 [arXiv:0710.5420 [astro-ph]].

[5] S. Palomares-Ruiz, Phys. Lett. B 665 (2008) 50 [arXiv:0712.1937 [astro-ph]].

[6] S. Desai et al. [Super-Kamiokande Collaboration], Phys. Rev. D 70 (2004) 083523 [Erratum-ibid. D 70 (2004)] 109901 [arXiv:hep-ex/0404025].

[7] M. Ackermann et al. [AMANDA Collaboration], Astropart. Phys. 24 (2006) 459 [arXiv:astro-ph/0508518]; G. Wikstrom [IceCube Collaboration], in proceedings of ICRC 2007 [in arXiv:0711.0353 [astro-ph], pages 135-138]; G. Lim [ANTARES Collaboration], in proceedings of SUSY07 [arXiv:0710.3685 [astro-ph]].

[8] A. Cervera, F. Dydak and J. Gomez Cadenas, Nucl. Instrum. Meth. A 451 (2000) 123; A. Cervera-Villanueva, Nucl. Phys. Proc. Suppl. 149 (2005) 201.

[9] J. Angle et al. [XENON Collaboration], Phys. Rev. Lett. 100 (2008) 021303 [arXiv:0706.0039 [astro-ph]]; Z. Ahmed et al. [CDMS Collaboration], arXiv:0802.3530 [astro-ph].

[10] H. S. Lee. et al. [KIMS Collaboration], Phys. Rev. Lett. 99 (2007) 091301 [arXiv:0704.0423 [astro-ph]]; E. Behnke et al. [COUPP Collaboration], Science 319, 933 (2008) [arXiv:0804.2886 [astro-ph]].

[11] M. Cirelli et al., Nucl. Phys. B 727 (2005) 99 [arXiv:hep-ph/0506298].

[12] M. Malek et al. [Super-Kamiokande Collaboration], Phys. Rev. Lett. 90 (2003) 061101 [arXiv:hep-ex/0209028].

[13] K. Griest and M. Kamionkowski, Phys. Rev. Lett. 64 (1990) 615; L. Hui, Phys. Rev. Lett. 86 (2001) 3467 [arXiv:astro-ph/0102349].

[14] M. Kaplinghat, L. Knox and M. S. Turner, Phys. Rev. Lett. 85 (2000) 3335 [arXiv:astro-ph/0005210].

[15] M. Kachelriess and P. D. Serpico, Phys. Rev. D 76, 063516 (2007) [arXiv:0707.0209 [hep-ph]];

N. F. Bell, J. B. Dent, T. D. Jacques and T. J. Weiler, arXiv:0805.3423 [hep-ph]; J. B. Dent, R. J. Scherrer and T. J. Weiler, arXiv:0806.0370 [astro-ph].

[16] K. Ichiki, M. Oguri and K. Takahashi, Phys. Rev. Lett. 93 (2004) 071302 [arXiv:astro-ph/0403164].

[17] Y. Gong and X. Chen, Phys. Rev. D 77 (2008) 103511 [arXiv:0802.2296 [astro-ph]].

[18] G. D. Mack, T. D. Jacques, J. F. Beacom, N. F. Bell and H. Yuksel, arXiv:0803.0157 [astro-ph].

[19] S. Palomares-Ruiz, in proceedings of Rencontres de Moriond EW 2008 [arXiv:0805.3367 [hep-ph]]. 